\title{
Spinnen aus Malaisefallen
}

\author{
Bodo von BROEN
}

\begin{abstract}
Spiders from Malaise traps. The spider fauna of the higher vegetation strata is by far less well investigated than the bulk of epigeic spiders. The present paper refers to spiders collected in Malaise traps that were used during an insect sampling program in Brandenburg (Germany) in 1992-1993. In spite of a rather unusual sampling technique for spiders, the species report presented might add to our knowledge useful data on arboricolous spiders.
\end{abstract}

Key words: Malaise traps for spider sampling

\section{EINLEITUNG}

Webspinnen, die höhere Strata der Vegetation als Lebensraum nutzen, sind wesentlich weniger intensiv untersucht worden als die epigäisch lebenden Vertreter. Ursachen dafür liegen vor allem in methodischen Schwierigkeiten. Ein besonderes Problem bietet die Untersuchung der höheren Stammregionen und des Kronenbereichs von Bäumen. Wertvolle Erkenntnisse über die Spinnenbesiedlung dieser Habitate verdanken wir grundlegenden Bemühungen von HESSE (1940), ENGELHARDT (1958), HÖREGOTT (1960), TURNBULL (1960), ZITNANSKA (1970), KLOMP \& TEERINK (1973). Diese Autoren näherten sich der Fragestellung durch Käscher- und Schüttelfänge im Bereich der Baumkronen oder durch das Absägen von Ästen oder ganzer Bäume, um zu einer quantitativen Erfassung der dort lebenden Spinnen zu kommen. Eine Kenntniserweiterung brachte der Einsatz von Baum-Photoeklektoren (ALBERT 1976), die vor allem am Stamm wandernde bzw. im Rindenbereich lebende Formen nachzuweisen gestatten. Den Rindenbewohnern hatWUNDERLICH(1982) eine besondere Untersuchung gewidmet, da die mitBaum-Photoeklektoren ermittelten Spinnenarten sowohl Kronenbewohner als auch Besiedler des Stammbereiches und natürlich auch Zufallsgäste sein können. Die Vernachlässigung dieses Aspekts hat Anlaß zu zahlreichen Fehlinterpretationen der Habitatwahl von Spinnen höherer Strata gegeben (vgl.WUNDERLICH 1982). - Ein indirekter Nachweis arboricoler Spinnen wird durch den 
Einsatz von Malaisefallen möglich, wie HAUGE \& MIDTGAARD (1986) eindrucksvoll zeigten. Die für den Insektenfang konzipierten Malaisefallen liefern als Beifang meistens eine geringe Spinnenausbeute, die im Regelfall vernachlässigt wird. Da sich darunter aber ein erheblicher Teil hypergäisch lebender Vertreter befindet, kann die Auswertung derartiger Fänge wertvolle Hinweise auf Arten geben, die mit Bodenfallen nicht oder nur zufällig nachgewiesen werden.

Bei entomofaunistischen Bestandsaufnahmen in Brandenburg kamen 1992-1993 neben anderen Methoden auch Malaisefallen zum Einsatz, deren Ausbeute eine Anzahl von Webspinnen enthielt. Ihre Bearbeitung erschien lohnend, weil mit dem Nachweis von Arten gerechnet werden konnte, über deren Vorkommen und Erscheinungszeit im hiesigen Gebiet wir weniger wissen als über die Präsenz und die Phänologie epigäisch lebender Spinnen.Die Ergebnisse der Auswertung werden in vorliegender Arbeit mitgeteilt.

\section{UNTERSUCHUNGSGEBIET UND METHODIK}

Untersuchungsgebiet ist das Biosphärenreservat Schorfheide-Chorin im Raum Eberswalde-Angermünde, das zu den wertvollsten schutzwürdigen Naturräumen Brandenburgs gehört. In diesem Gebiet laufen Erhebungen zur Aktualisierung derRoten Listen gefährdeter Tiere, die eine Überprüfung der umfangreichen Basisdaten für verschiedene Arthropodengruppen erfordern.

Die neben Barberfallen für die Insektenbestandsaufnahme eingesetzten Malaisefallen werden nachfolgend mit den Kürzeln St1 und St3 - St9 gemäß den Untersuchungsstandorten zitiert.

\section{Standortcharakterisierung}

St1: Kleiner Rummelsberg bei Brodowin. Es handelt sich um einen aus Grundmoränenmaterial aufgeschütteten ovalen Hügel (sog. Drumlin) mit Trockenrasenbewuchs (u.a. Stipa capillata), der steil aus dem Parsteiner Zungenbecken aufragt ( $80 \mathrm{~m}$ ü.NN). Die Malaisefalle stand am nordexponierten teilweise verbuschten Hang.

St3: Kernberge bei Klein-Ziethen, Kalk- bzw. Sandtrockenrasenhang am Serwester See mit Schwarzdornhecke und Eschen-Ahorn am Hangfuß. Die Falle stand am Hangfuß im Gebüschsaum. 
St4: Grumsiner Forst bei Louisenfelde, naturnaher Buchenwald mit erlenbruchartiger Senke (eutrophes Kleingewässer) in einer Kernzone des Biosphärenreservates. Fallenstandort an der Grenze zwischen Buchenaltholz und Erlenbruch.

St5: Kiefernmischwald nördlich Golzow mit Anschluß an Ackerflächen, Übergangsbereich mit Lesesteinriegel und Magerrasenflächen. Fallenstandort am Randsaum des Gehölzes.

St6: Kiefernforst mit Laubholzunterwuchs nördlich Britz mit Anschluß an Ackerflur; Übergangsbereich Magerrasen mit starker Verqueckung. Fallenstandort am Randsaum des Waldes.

St7: Ackerfläche im Anschluß an St6, 1992 mit Mais bestellt, 1993 brachliegend. Fallenstandort Brachfläche.

St8: Soll in der Feldflur bei Groß-Ziethen: landschaftstypische Sollbildung von ca. 0,5 ha mit stark abfallenden Rändern und Weidenbewuchs; Wassertiefe im Mai 1993 etwa 0,5 m. Fallenstandort im Randbereich der Kernzone.

St9: Kesselmoor westlich Chorin innerhalb eines Buchenhangwaldes; Moorfläche ca. 2 ha. Fallenstandort an der Grenze des Feuchtbereiches.

Der Wechsel der Fanggefäße in den Malaisefallen erfolgte in wöchentlichem Abstand vom 8.4.1993 bis 4.11.1993. Im Jahre 1992 wurden lediglich die Standorte St1, St3 und St4 mit Malaisefallen untersucht. Die Leerung erfolgte unregelmäßig.

Der Artennachweis erfolgt nach Familienzugehörigkeit. Gattungen und Arten werden in alphabetischer Reihenfolge genannt. Es bedeuten: $\mathbf{x}, \mathbf{y} / \mathbf{z}=$ Männchen,Weibchen/inad. Tiere

Auf Angaben zur Phänologie wird verzichtet, da die Zahl der Exemplare pro Art zu gering ist und für die meisten Spezies hinreichende Kenntnisse vorliegen. Statt dessen wird für jede Art hinter der Namensnennung die Stratumzugehörigkeit, soweit möglich, genannt. Dafür gelten folgende Ziffern:

0: hypogäisch lebend (z.B. unter Steinen)

1: epigäisch auf der Erdoberfläche bzw. in der Streu

2: auf oder zwischen den Pflanzen der Krautschicht

3: in der Strauchschicht oder unteren Zweigen der Bäume

3a: Arten der Baumrinde und Netzbauer am Stamm

4: in höheren Baumregionen lebend

5: in der Kronenregion von Bäumen lebend. 
Diese Einteilung ermöglicht nicht mehr als eine grobe Zuordnung, da Übergänge zwischen 0 und 1, 1 und 2 sowie 3-5 bestehen. Aufgrund unserer unzureichenden Kenntnisse bestehen insbesondere hinsichtlich der hypergäisch lebenden Formen ( vor allem der Arboricolen) enorme Unsicherheiten.

ERGEBNISSE (ARTENNACHWEISE)

Taxon

Stratum

St: Anzahl

FAMILIE SEGESTRIIDAE

Segestria senoculata (LINNÉ,1758)

$3 a$

St5:

1,0

FAMILIE MIMETIDAE

Ero furcata (VILLERS,1789)

$2-4$

St5:

0,1

FAMILIE THERIDIIDAE

Achaearanea tepidariorum (C.L.KOCH,1841)

Enoplognatha ovata (CLERCK,1757)

3-4

$2-4$

Neottiura bimaculata (LINNÉ, 1767)

2

Theridion impressum L.KOCH,1881

$2-3$

Theridion pinastriL.KOCH,1872

Theridion tinctum (WALCKENAER,1802)

Theridion varians $\mathrm{HAHN}, 1833$

2

St8: $\quad 1,0$

St3: $\quad 1,0$

St4: $\quad 15,11 / 1$

St9: $\quad 2,1$

St5: $\quad 1,0$

St8: $\quad 0,2$

St7: $\quad 6,0$

St8: $\quad 2,0$

St7: $\quad 0,1$

St9: $\quad 1,0$

St1: $\quad 0,0 / 1$

St6: $\quad 1,1$

St8: $\quad 3,0$

St9: $\quad 2,0$

FAMILIE LINYPHIIDAE (ERIGONINAE)

Araeoncus humilis (BLACKWALL,1841)

St1: $\quad 0,1$

St3: $\quad 1,0$

St5: $\quad 2,1$

St6: $\quad 1,1$

Ceratinella brevis (WIDER,1834)

St6: $\quad 0,1$

Erigone atra BLACKWALL, 1833

1

St3: $\quad 0,2$

St4: $\quad 1,0$

St5: $\quad 1,0$

St6: $\quad 0,1$

St8: $\quad 0,1$ 
Pelecopsis radicicola (L.KOCH,1872)

Savignya frontata (BLACKWALL, 1833)

Tapinocyba insecta (L.KOCH,1869)

Tapinocyba praecox (O.P.-CAMBRIDGE,1873)

Thyreosthenius parasiticus (WESTRING,1851)

Tiso vagans (BLACKWALL, 1834)

Walckenaeria monoceros (WIDER,1834)

FAMILIE LINYPHIIDAE (LINYPHIINAE)

Bathyphantes gracilis (BLACKWALL, 1841)

Diplostyla concolor (WIDER,1834)

Lepthyphantes angulipalpis (WIDER,1851)

Lepthyphantes decolor (WESTRING,1862)

Lepthyphantes flavipes (BLACKWALL,1854)

Lepthyphantes mengei KULCZYNSKI,1887

Lepthyphantes tenuis (BLACKWALL,1852)

Linyphia hortensis SUNDEVALL, 1830

Linyphia triangularis (CLERCK,1757)

Meioneta rurestris (C.L.KOCH,1836)

Neriene clathrata (SUNDEVALL,1830)

Porrhomma microphthalmum (O.P.-CAMBR.,1871)

$\begin{array}{ll}\text { St1: } & 1,0 \\ \text { St5: } & 1,0 \\ \text { St6: } & 1,0 \\ \text { St7: } & 1,0 \\ \text { St6: } & 0,2 \\ \text { St4: } & 1,0 \\ \text { St4: } & 2,3 \\ \text { St3: } & 1,0 \\ \text { St5: } & 0,1 \\ \text { St4: } & 0,1 \\ \text { St5: } & 0,1 \\ \text { St6: } & 0,1\end{array}$

St4:

St5:

1,1

1,0

1-2

St8:

1,0

1-2

St3:

1,0

1

1-3

1

1

1

1-3

1-2

St5:

1,0

St3: $\quad 0,1$

St3: $\quad 0,1$

St9:

1,0

St3:

St8:

2,2

1,1

St5:

1,0

St1: $\quad 0,0 / 1$

St4: $\quad 2,2 / 3$

St5: $\quad 3,1 / 3$

St6: $\quad 1,1$

St8: $\quad 1,0$

St9: $\quad 1,4 / 1$

1

St1:

St3:

St5:

St6:

St8:

1,1

1,0

4,3

0,1

0,2

1-2

St8:

St9:

$0,0 / 1$

1,0

St5: 
FAMILIE METIDAE

Metellina segmentata (CLERCK,1757)

2-4

St1:

1,0

St3: $\quad 1,0$

St4: $\quad 1,0$

St5: $\quad 2,0$

St9: $\quad 4,0$

Zygiella atrica (C.L.KOCH,1845)

St1:

1,0

FAMILIE TETRAGNATHIDAE

Pachygnatha clerckiSUNDEVALL,1823

Pachygnatha degeeriSUNDEVALL,1830

Tetragnatha montana SIMON, 1874

Tetragnatha nigrita LENDL, 1886

Tetragnatha obtusa C.L.KOCH,1837

$\begin{array}{rll}1 & \text { St9: } & 0,1 \\ 1 & \text { St3: } & 1,1 \\ 3 & \text { St1: } & 0,1 \\ 2-3 & \text { St1: } & 1,0 \\ 2-5 & \text { St9: } & 1,1\end{array}$

FAMILIE ARANEIDAE

Araneus diadematus CLERCK,1757

Araniella cucurbitina (CLERCK,1757)

2-3 St3: $\quad 2,0$

2-4 St3: $\quad 2,0$

St9: $\quad 1,0$

Araniella opisthographa (KULCZYNSKI,1905)

2-4

St1:

1,0

St3: $\quad 1,0$

Argiope bruennichi (SCOPOLI,1772)

2

St3:

1,0

Larinioides cornutus (CLERCK,1757)

2

St1:

3,2

Larinioides patagiatus (CLERCK,1757)

3-4

Mangora acalypha (WALCKENAER, 1802)

St1: $\quad 45,5$

St3: $\quad 1,0$

St1: $\quad 0,0 / 1$

St3: $\quad 0,0 / 1$

FAMILIE LYCOSIDAE

Pardosa prativaga (L.KOCH,1870)

1

St3:

1,0

FAMILIE PISAURIDAE

Pisaura mirabilis (CLERCK,1757)

St6: $\quad 0,0 / 1$

St9: $\quad 0,1 / 1$

FAMILIE AGELENIDAE

Agelena labyrinthica (CLERCK,1757)

St6:

0,1

FAMILIE DICTYNIDAE

Dictyna pusilla THORELL, 1856

St9:

0,1 
FAMILIE ANYPHAENIDAE

Anyphaena accentuata (WALCKENAER, 1802)

1-4 St3: $\quad 0,0 / 1$

St4: $\quad 0,0 / 10$

St9: $\quad 0,0 / 2$

FAMILIE CLUBIONIDAE

Clubiona brevipes BLACKWALL, 1841

2-3 St3: $\quad 1,0$

St7: $\quad 0,1$

St9: $\quad 1,0$

Clubiona frutetorum L.KOCH,1867

2-3 St1: $\quad 8,4$

St7: $\quad 1,0$

Clubiona genevensis L.KOCH,1867

0-4 St3: $\quad 1,0$

St5: $\quad 1,0$

St9: $\quad 0,1$

Clubiona germanica THORELL, 1870

Clubiona neglecta O.P.-CAMBRIDGE, 1862

Clubiona pallidula (CLERCK,1757)

1-4 St8: $\quad 1,0$

1-4 St8: 0,1

3-4 St1: 3,5

St3: $\quad 0,1$

St4: $\quad 1,2$

St5: $\quad 3,0$

St6: $\quad 3,1$

St8: $\quad 1,1$

St9: $\quad 0,2$

Clubiona phragmitis C.L.KOCH, 1843

1-2 St3: $\quad 1,0$

St8: $\quad 5,2$

Clubiona terrestris WESTRING, 1861

St4: $\quad 4,3$

St8: $\quad 1,0$

FAMILIE GNAPHOSIDAE

Drassodes lapidosus (WALCKENAER,1802)

Poecilochroa conspicua (L.KOCH,1866)

0-1 St3: $\quad 0,1$

St6: $\quad 0,1$

2-3 St6: $\quad 0,1$

\section{FAMILIE PHILODROMIDAE}

Philodromus aureolus (CLERCK,1757)

Philodromus cespitum (WALCKENAER,1802)

Philodromus collinus C.L.KOCH, 1835

Philodromus disparWALCKENAER,1826

2-4 St6: $\quad 1,1$

St9: $\quad 0,1$

2-4 St1: $\quad 11,3$

St3: $\quad 2,2$

St8: $\quad 2,0$

St9: $\quad 0,1$

1-4 St1: $\quad 1,0$

St3: $\quad 2,0$

St6: $\quad 1,0$

1-4 St5: $\quad 1,0$ 
Philodromus rufus WALCKENAER, 1826

Tibellus oblongus (WALCKENAER,1802)
$2-3$

2
St5:

St6:

St3:

St6:

St8:

$\begin{array}{rll}3 a & \text { St6: } & 0,1 \\ 2-4 & \text { St9: } & 0,1 \\ 1 & \text { St3: } & 0,1 \\ 1-2 & \text { St4: } & 1,0 \\ 1 & \text { St1: } & 1,0 \\ & \text { St3: } & 3,1\end{array}$

FAMILIE SALTICIDAE

Ballus chalybeius (WALCKENAER,1802)

Heliophanus cupreus (WALCKENAER,1802)

Heliophanus flavipes HAHN, 1832

Salticus cingulatus (PANZER,1797)

Salticus scenicus (CLERCK,1757)

Salticus zebraneus (C.L.KOCH,1837)

$\begin{array}{lll}1-3 & \text { St4: } & 0,1 \\ & \text { St5: } & 0,2 \\ 2-3 & \text { St3: } & 1,0 \\ 1-4 & \text { St1: } & 0,1 \\ 2-4 & \text { St1: } & 1,8 \\ 3-5 & \text { St5: } & 0.1 \\ 3-4 & \text { St5: } & 1,1 \\ & \text { St6: } & 2,1 \\ & \text { St7: } & 1,0\end{array}$

\section{DISKUSSION}

Erwartungsgemäß rekrutieren sich die nachgewiesenen Spezies zu einem hohen Prozentsatz aus den Familien der Theridiiden, Clubioniden, Philodromiden und Thomisiden. In diesen Taxa finden wir zahlreiche Strauch- und Baumbewohner, die in Bodenfallen selten und eher zufällig auftreten. Typische Vertreter sind:

Theridiidae: Achaearanea tepidariorum, Neottiura bimaculata, Theridion impressum, Theridion neglectum, Theridion pinastri, Theridion varians; Clubionidae: Clubiona brevipes, Clubiona frutetorum, Clubiona genevensis, Clubiona germanica, Clubiona pallidula;

Philodromidae: alle Philodromus-Arten;

Thomisidae: Coriarachne depressa, Misumena vatia.

Von den nachgewiesenen Salticiden sind Ballus chalybeius und die Heliophanus-Arten als Besiedler der Baum- und Strauchschicht bekannt. Das Auftreten der 3 einheimischen Salticus-Arten kann ebenfalls nicht 
überraschen. Sie werden als tagaktive Jäger sehr häufig an vertikalen besonnten Flächen jeder Art, auch an Baumstämmen, gefunden.

Im Gegensatz zu den vorgenannten Artengruppen überrascht die relativ große Zahl von Linyphiiden, die als Bodenbewohner bekannt sind. Diese Tiere gerieten mit großer Wahrscheinlichkeit als Aeronauten in den Fangbereich der Malaisefallen, wie etwa Araeoncus humilis, Erigone atra, Oedothorax apicatus, Savignya frontata, Tapinocyba praecox, Walckenaeria monoceros. Das Gleiche gilt mit gewisser Einschränkung für die Mehrzahl der Baldachinspinnen, wenn man die Angaben über die Stratumpräferenz betrachtet.Auffällig an dem ausgewerteten Material ist das Fehlenobligater Baumbewohner wie Entelecara, Agyneta innotabilis oder bestimmter Lepthyphantes-Spezies.

Einige der nachgewiesenen Arten verdienen Interesse aus faunistischen und autökologischen Gründen:

Porrhomma microphthalmum: Die im Untersuchungsgebiet auf Äckern und Brachflächen nicht seltene Linyphiide ist wahrscheinlich durch Winddrift in den Fallenbereich geraten. Die Kenntnisse über die Lebensweise der einheimischen Porrhomma-Arten sind allerdings so dürttig, daß Angaben über die Habitatpräferenz mit Zurückhaltung betrachtet werden müssen.

Clubiona germanica: Der vorliegende Nachweis eines Männchens (28.10.1993) ist der einzige Beleg im weiteren Untersuchungsgebiet während des Zeitraums der Studien. Die Art wird selten zitiert; über ihre Habitatansprüche besteht offenbar Unklarheit. WEISS (1987) ordnet sie für sein Untersuchungsgebiet den Faunenelementen von Bachufern und Auweidengesellschaften zu. Der hiesige Fund spricht ebenfalls für die Besiedlung feuchter Standorte. In der Roten Liste für Bayern ist C.germanica sogar als gefährdet eingestuft!

Poecilochroaconspicua: Nachweise dieserGnaphoside sind eineSeltenheit. Die Tiere leben in der Kraut- und Strauchschicht, was den Fund in der Malaisefalle erklärt. Ihr Vorkommen im Untersuchungsgebiet ist erstmalig belegt.

Philodromus rufus: $P$. rufus wird allgemein seltener als P.cespitum, $P$. collinus und $P$.dispargenannt. Seine Verbreitung im hiesigen Gebiet ist genauer zu untersuchen. Er tritt hier syntopisch mit den vorgenannten Arten in der Krautschicht und der Gebüschzone an Waldrändern auf.

Coriarachne depressa: Das Tier ist ein typischer Rindenbewohner, doch scheinen zumindestens die Männchen einen erheblichen Aktivitätsradius zu haben. Auch HAUGE \& MIDTGAARD (1986) verweisen auf den Fang zweier Männchen der Art in Malaisefallen. KNOFLACH \& BERTRANDI 
(1993) melden den Nachweis der Art aus Klopffängen an Juniperus und Pinus, darunter auch ein Weibchen.

\section{SCHLUSSFOLGERUNG}

Die vorliegenden Befunde bestätigen die Möglichkeit, Bestandserhebungen über die Araneenfauna eines Gebietes im Hinblick auf hypergäischlebende Arten durch Auswertung des Beifanges aus Malaisefallen zu bereichern. Dadurch sind u.a. Daten über silvicole Arten höherer Strata zu gewinnen, deren Häufigkeit nicht sicher beurteilt werden kann. Die Schwierigkeiten, die sich daraus bei der Festlegung inres Gefährdungsgrades ergeben, sind bekannt. Erkenntnisse über die Habitatpräferenz bzw.über das bevorzugte Stratum der Vegetation lassen sich mit Malaisefallen allerdings nicht sammeln.

\section{LITERATUR}

ALBERT, R. (1976): Zusammensetzung und Vertikalverteilung der Spinnenfauna in Buchenwäldern des Solling. - Faun.-Ökol. Mitt.5: 65-80; Kiel

ENGELHARDT, W. (1958): Untersuchungen über Spinnen aus Fichtenwipfeln. - Opuscula Zool.17: 1-9; München

HAUGE, E. \& F. MIDTGAARD (1986): Spiders (Araneae) in Malaise traps from two islands in the Oslofjord, Norway. - Fauna norv. Ser. B.33: 98-102; Oslo

HESSE, E. (1940): Untersuchungen an einer Kollektion Wipfelspinnen. -S.-B. Ges. naturforsch. Freunde 193: 350-363; Berlin

HÖREGOTT, H. (1960): Untersuchungen über die qualitative und quantitative Zusammensetzung der Arthropodenfauna in den Kiefernkronen. - Beitr. Ent. 10: 891-916; Berlin

KLOMP, H. \& B.J.TEERINK (1973): The density of the invertebrate summer fauna on the crowns of pine trees, Pinus sylvestris, in the central parts of the Netherlands. - Beitr. Ent. 23: 325-340; Berlin

KNOFLACH, B. \& F. BERTRANDI (1993): Spinnen (Araneida) aus Klopffängen an Juniperus und Pinus in Nordtirol. - Ber. nat.-med. Verein Innsbruck 80: 295-302; Innsbruck

TURNBULL, A.L. (1960): The spider population of a stand of oak (Quercus roburL.) in Wytham Wood, Berks., England. - Can.Entomol. 92: 110-124

WEISS, I. (1987): Araneele zonei colinare din sudul Transilvaniei. Un conspect al datelor faunistice si ecologice. (Arachnida: Araneae). - Anuar Complexul Muzeal Sibiu 1:297-318 WUNDERLICH, J. (1982): Mitteleuropäische Spinnen (Araneae) der Baumrinde. - Z.ang.Ent. 94: 9-21

ZITNANSKA, O. (1970): Arachnofauna of Querco-Carpinetum. - AtBABRes. ProjectBAB (IBP) Progr.Rep. 1: 165-168; Bratislava

\section{Dr. Bodo von BROEN, Fürstenwalder Straße 17, D-10243 Berlin}

